\title{
Geographical heterogeneity of multidrug-resistant tuberculosis in Georgia, January 2009 to June 2011
}

H E Jenkins (jenkins.helen@gmail.com) ${ }^{1,2}$, M Gegia3 ${ }^{3}$ J Furin4, I Kalandadze5, U Nanava3, T Chakhaia ${ }^{3}$, T Cohen ${ }^{1,6}$

1. Brigham and Women's Hospital, Boston, USA

2. Harvard Medical School, Boston, USA

3. The National Center for TB and Lung Disease, Tbilisi, Georgia

4. TB Research Unit, Case Western Reserve University School of Medicine, Cleveland, Ohio, USA

5. David Agmashenebeli University of Georgia, Tbilisi, Georgia

6. Harvard School of Public Health, Boston, US

Citation style for this article:

Jenkins HE, Gegia M, Furin J, Kalandadze I, Nanava U, Chakhaia T, Cohen T. Geographical heterogeneity of multidrug-resistant tuberculosis in Georgia, January 2009 to June 2011. Euro Surveill. 2014;19(11):pii=20743. Available online: http://www.eurosurveillance.org/ViewArticle.aspx?Articleld=20743

Article submitted on 05 June 2013 / published on 20 March 2014

In 2011, Georgia, in the Caucasus, reported that $11 \%$ of new and $32 \%$ of previously treated tuberculosis (TB) cases nationally had multidrug-resistant TB (MDR-TB). To help understand the mechanisms driving these high risks of drug-resistance and plan for targeted interventions, we identified geographical variability in the MDR-TB burden in Georgia and patient-level MDR-TB risk factors. We used routinely collected surveillance data on notified TB cases to estimate the MDR-TB incidence/100,000 people and the percentage of TB cases with MDR-TB for each of 65 districts and regression modelling to identify patient-level MDR-TB risk factors. 1,795 MDR-TB cases were reported (January 2009-June 2011); the nationwide notified MDR-TB incidence was $16.2 / 100,000$ but far higher $(837 / 100,000)$ in the penitentiary system. We found substantial geographical heterogeneity between districts in the average annual MDR-TB incidence/100,000 (range: 0.0-5.0 among new and 0.0-18.9 among previously treated TB cases) and the percentage of TB cases with MDR-TB (range: $0.0 \%-33.3 \%$ among new and $0.0 \%-75.0 \%$ among previously treated TB cases). Among treatment-naïve individuals, those in cities had greater MDR-TB risk than those in rural areas (increased odds: $43 \%$; $95 \%$ confidence interval: $20 \%-72 \%)$. These results suggest that interventions for interrupting MDR-TB transmission are urgently needed in prisons and urban areas.

\section{Introduction}

In 2011, there were an estimated 8.7 million newly infected cases of tuberculosis (TB) worldwide and 1.4 million deaths attributed to TB [1]. The appearance and spread of forms of Mycobacterium tuberculosis that are resistant to drugs in the standardised TB treatment regimen are threats to effective TB control. People suffering from multidrug-resistant TB (MDR-TB, i.e. TB that is resistant to at least isoniazid and rifampicin) require longer treatment with second-line drugs (SLD) that are more expensive and more toxic than those in the standard TB drug regimen; even in settings where individuals receive the best available care, poor outcomes are common [2].

Global estimates indicate that $3.7 \%$ of new TB cases and $20 \%$ of previously treated TB cases have MDR-TB [1], but these averages mask substantial geographical heterogeneity in risk. Countries of the eastern part of the World Health Organization (WHO) European Region have reported percentages of TB cases with MDR-TB several times higher than countries elsewhere in the world $[1,3]$. Georgia is a country of approximately 4.5 million people [4] located in the Caucasus and, like many countries in this region, it is experiencing a MDR-TB crisis. In response to a growing regional appreciation for the severity of this issue, Georgia made further investment in their commitment to provide universal access to diagnosis and treatment for drug-resistant TB [5] and begin routine surveillance to monitor drug resistance in 2006. As of 2011, Georgia was one of only six countries (among the 27 high burden MDR-TB countries) to have routine TB surveillance in place (i.e. nationwide, continuous, real-time notifications of all diagnosed drug resistant TB cases as opposed to sub-national reporting and/or periodic surveys) [1]. In $2011,11 \%$ of notified new TB cases and $32 \%$ of notified previously treated TB cases in Georgia had MDR-TB and the national estimated TB incidence rate was $125 / 100,000$ [1].

Statistics on MDR-TB burden are usually reported at the country level and few countries have sufficient detailed spatial resolution of data to examine local heterogeneity of MDR-TB burden [6]. Previous work has indicated that even in countries where TB patients have a very high overall risk of MDR-TB, the spatial variation in this risk can be dramatic, indicating potential opportunities for prioritising earliest responses and confirmatory studies to areas deemed at highest risk [6-8]. Here, we present spatial analyses of MDR-TB risk and incidence across Georgia in an attempt to identify 
areas of relative high risk and/or incidence of MDR-TB among both new and previously treated TB cases. We also evaluate patient-level risk factors for MDR-TB amongst these patients.

\section{Methods}

\section{Data sources}

We analysed two TB surveillance databases that contained information on: (i) all TB cases notified in Georgia between January 2009 and December 2011 (database 1) and (ii) all patients that were hospitalised and initiated on SLD in Georgia between January 2009 and December 2011 (database 2).

In Georgia, all suspected TB cases based on clinical findings and chest radiography receive sputum smear microscopy at their local TB facility. All sputum samples, regardless of their microscopy findings, are then transported to the closer of two laboratories in the cities of Tbilisi or Kutaisi for culture testing. A cold chain is maintained and transportation time does not exceed 24 hours. Both laboratories have passed External Quality Assurance conducted by the Supranational Reference Laboratory in Antwerp, Belgium and are the only two laboratories in Georgia that perform TB culture testing. TB diagnosis is confirmed by positive microscopy and/ or culture testing; both tests are required for all TB suspects under the national TB policy. Drug susceptibility testing (DST) is required under national policy for all culture positive sputum samples and is carried out only in the laboratory in Tbilisi. Culture and DST are done by conventional Lowenstein-Jensen solid media and/or broth-based culture methods using the MGIT 960 system (BD, Sparks, MD, USA) [9].

When a TB case is notified, a paper form is completed by the attending physician containing demographic data on the case (including information such as age, sex, previous treatment status and previous detention status). These forms are sent to the regional database manager and entered into the online TB notifications database (database 1). When culture and DST results are available, these are sent in paper forms to the database manager in Tbilisi and entered into the same online TB database.

All TB patients in Georgia are initially hospitalised and remain there until their smear microscopy results are negative and their clinical condition is stable. If patients have MDR-TB or polydrug-resistant TB confirmed by DST or do not receive culture and/or DST but are suspected MDR-TB cases such as household contacts of an MDR-TB case, they are hospitalised and initiated on SLD. Once these patients have started treatment, they are entered onto a second TB database (database 2). The average length of stay in hospital is two months for non-MDR-TB patients and three to four months for patients on SLD.
Georgia achieved universal access to TB diagnosis and treatment by the end of 2009. Since then, all notified TB (non-MDR-TB and MDR-TB) patients have had access to treatment as per WHO recommendations [10].

For our spatial analysis, each patient is indexed by the TB outpatient facility located within their district/city of residence (as defined in the National Census [4], a total of 72 districts and cities); these districts/cities are further aggregated into 12 administrative regions. There are seven facility codes within the city of Tbilisi, which we grouped together for our analysis. All patients diagnosed within the penitentiary system were attributed to the penitentiary system and were excluded from the spatial analysis. In addition, the region Abkhazia (consisting of 6 districts) and the district Java do not notify TB patients in either TB surveillance database. These seven districts were excluded and therefore the analysis focused on a total of 65 districts/cities. Five of the districts are classed as 'cities' and therefore our definition of 'urban areas' included these five cities. All other areas were classed as 'rural areas'.

\section{Statistical analysis}

Estimation of the absolute number of multidrugresistant tuberculosis notifications and multidrugresistant tuberculosis incidence

We aimed to report the number of MDR-TB cases that were notified between 2009 and 2011 and confirmed through DST. In practice, not all culture and DST results were entered in the TB notifications database (database 1 ); $33.5 \%$ of cases recorded in database 1 did not have any information on culture results recorded in that database. Therefore, we used database 2 (hospitalisations for second-line treatment) as our primary source to calculate the number of MDR-TB notifications (93.4\% of cases in database 2 had sufficient DST to diagnose or rule-out MDR-TB). From this database, we extracted the number of MDR-TB cases diagnosed and confirmed through DST. However, since it is a database of cases that initiated treatment between January 2009 and December 2011, some cases that were diagnosed in late 2011 and had not initiated treatment by the end of 2011 were not yet entered in this database. Hence, to minimise such errors, we included cases that were diagnosed between January 2009 and June 2011 inclusive. Additionally, since this database included all those cases that were hospitalised, it excluded some MDR-TB patients who were not hospitalised (for example, due to death or default prior to hospitalisation or due to lack of available treatment during 2009 before universal access became available) or were missing from database 2 due to data entry errors. Therefore, we also identified all DST-confirmed MDR-TB cases that were recorded between January 2009 and June 2011 in the TB notifications database (database 1) that were not found in the SLD hospitalisations database (database 2) and included these in our count of MDR-TB diagnoses. If one individual had two TB episodes during the study period, these would be considered as 
two incident cases. This was to correctly capture the burden placed on TB services since each incident case needs to be diagnosed and placed on treatment each time.

To estimate average annual MDR-TB incidence per 100,000 population, we divided our count of MDR-TB diagnoses by the population of Georgia in the same time period as reported in the National Census [4]. The most recent National Census was conducted in 2002. However, population numbers are recalculated annually using methods such as telephone interviews in order to fully account for variation in population changes at a local level and events that have resulted in substantial population movements. In addition to overall incidence, we estimated incidence rates by age and sex. We also estimated the average annual incidence of MDR-TB per 100,000 population for each district. All measures were estimated for new TB cases (i.e. those who have either previously received treatment for less than one month in total or never) and previously treated TB cases (i.e. those who have previously received one month or more TB treatment) separately [11]. Separate analyses of these two groups is important since resistance among new cases is the result of transmission of MDR-TB whereas resistance among previously treated cases may reflect either transmitted resistance or resistance acquired during previous exposure to TB drugs.

Estimation of the percentage of tuberculosis cases with multidrug-resistant tuberculosis

While measures of incidence provide information on the resource needs for programmes, measures of risk also provide useful additional information as they indicate the probability that individual patients diagnosed with disease will harbour an MDR-TB infection. To produce valid estimates of the percentage of TB cases that had MDR-TB, we needed to ensure that we estimated this risk from a population in whom DST was systematically employed without regard to actual risk of drug-resistance. Since, during the period of this analysis, DST was a routinely employed test, we used data extracted from the TB notifications database (database 1) under the assumption that there was no systematic bias in terms of risk of resistance between those TB cases that did or did not have data on DST results. We also estimated the percentage of TB cases that had MDR-TB for each district using database 1 . All measures were estimated for new and previously treated TB cases separately.

Patient-level risk factors for multidrug-resistant tuberculosis

We developed logistic regression models (for new and previously treated cases separately) to identify risk factors associated with MDR-TB diagnosis. As we did when estimating the percentage of TB cases with MDR-TB, we used only the data from the TB notifications database (database 1) under the assumption that there was no systematic bias in the availability of culture and DST results in each group (i.e. cases (confirmed MDR-TB
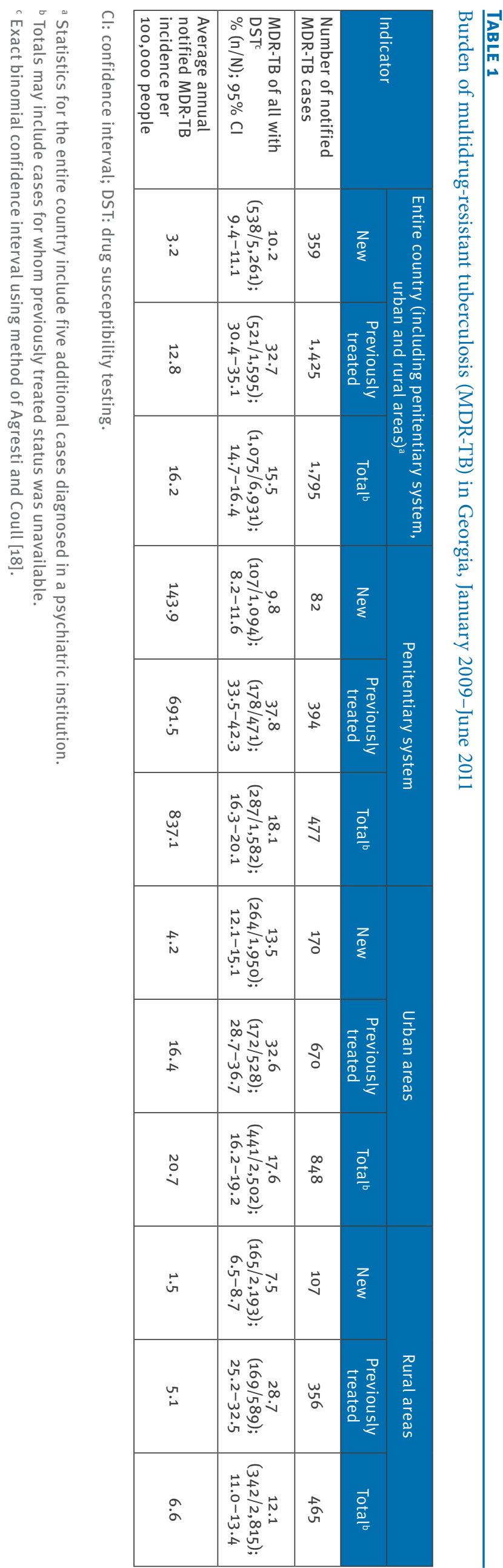
Age and sex-adjusted rates of notified multidrug-resistant tuberculosis (MDR-TB) incidence per 100,000 people, Georgia, January 2009-June 2011

\begin{tabular}{|c|c|c|c|c|c|c|c|c|c|}
\hline \multirow{3}{*}{$\begin{array}{l}\text { Age group } \\
\text { (years) }\end{array}$} & \multicolumn{9}{|c|}{ MDR-TB } \\
\hline & \multicolumn{3}{|c|}{$\begin{array}{l}\text { Among new TB cases } \\
\qquad(n=359)\end{array}$} & \multicolumn{3}{|c|}{$\begin{array}{l}\text { Among previously treated TB cases } \\
\qquad(n=1,425)\end{array}$} & \multicolumn{3}{|c|}{$\begin{array}{l}\text { Among all TB cases } \\
\qquad(n=1,795)\end{array}$} \\
\hline & Males & Females & Total & Males & Females & Total & Males & Females & Total \\
\hline $0-4$ & 0.9 & 1.3 & 1.1 & 1.2 & 0.0 & 0.6 & 2.0 & 1.3 & 1.7 \\
\hline $5-14$ & 0.8 & 0.8 & 0.8 & 0.8 & 0.5 & 0.6 & 1.7 & 1.4 & 1.5 \\
\hline $15-24$ & 6.2 & 2.1 & 4.2 & 18.5 & 6.9 & 12.8 & 24.9 & 9.1 & 17.1 \\
\hline $25-34$ & 11.4 & 2.5 & 6.9 & $43 \cdot 9$ & 9.9 & 26.8 & $55 \cdot 5$ & 12.5 & 33.9 \\
\hline $35-44$ & 7.6 & 0.6 & 4.0 & 44.2 & 5.0 & 23.8 & 52.0 & 5.6 & 27.8 \\
\hline $45-54$ & $5 \cdot 5$ & 1.3 & 3.2 & 27.9 & 2.9 & $14 \cdot 5$ & 33.9 & 4.2 & 18.0 \\
\hline $55-64$ & 3.0 & 1.0 & 1.9 & 20.0 & 2.0 & 10.1 & 23.0 & 2.9 & 12.0 \\
\hline$>64$ & 2.5 & 0.7 & 1.4 & $4 \cdot 7$ & 2.0 & 3.0 & $7 \cdot 3$ & 2.8 & $4 \cdot 5$ \\
\hline Total & 5.4 & 1.3 & 3.2 & 22.6 & 4.1 & 12.9 & 28.1 & 5.5 & 16.2 \\
\hline
\end{tabular}

TB: tuberculosis.

cases) and controls (cases confirmed without MDR-TB)). A full model was constructed including all potential explanatory variables to obtain fully adjusted odds ratios. Potential explanatory variables that we examined included demographic data (e.g. age and sex), socio-economic data (e.g. occupation and previous detention status) and TB-related data (e.g. previous TB treatment outcome and disease location). A backwards elimination method was used to identify variables that were statistically significantly associated with MDR-TB diagnosis. In addition, any non-statistically significant variable which, on removal, altered other parameter estimates substantially $(>10 \%)$ remained in the model to ensure full adjustment for confounding.

\section{Results}

\section{Burden of multidrug-resistant tuberculosis in Georgia}

Between January 2009 and June 2011, we identified 1,795 incident cases of MDR-TB confirmed through DST (Table 1) (1,370 from database 2 and an additional 425 were identified in database 1). Average annual MDR-TB notified incidence was 16.2 per 100,000 (3.2 new MDR-TB cases per 100,000 and 12.8 previously treated MDR-TB cases per 100,000). Incidence rates varied substantially by age and sex with the highest rates in men aged between 25 and 34 years (average of 55.5 annually per 100,000 , Table 2 ). The estimated percentage of TB cases with MDR-TB was $15.5 \%$ (95\% confidence interval (CI): $14.7 \%-16.4 \%$ ); an estimated $10.2 \%$ of new and $32.7 \%$ of previously treated TB cases had MDR-TB (Table 1). While the percentages of TB cases diagnosed in the penitentiary system with MDR-TB were similar to those in the civilian population, the incidence of notified MDR-TB was considerably higher at 837 cases per 100,000 ( 477 cases total, representing $27 \%$ of all MDR-TB notifications in Georgia) (Table 1).

\section{Geographical heterogeneity in multidrug-} resistant tuberculosis risk and incidence There was substantial variation in the MDR-TB burden by district. The incidence of notified MDR-TB per 100,000 varied from 0.0 to 5.0 for new cases and from 0.0 to 18.9 for previously treated cases (Figure 1). The percentage of TB cases with MDR-TB varied from $0.0 \%$ to $33.0 \%$ among new TB cases and from $0.0 \%$ to $75.0 \%$ among previously treated TB cases (Figure 2).

Both the MDR-TB incidence per 100,000 and the percentage of TB cases with MDR-TB were higher in urban areas than in rural areas. MDR-TB incidence in urban areas was $20.7 / 100,000$ compared with $6.6 / 100,000$ in rural areas and the percentage of TB cases with MDR-TB in urban areas was $17.6 \%$ compared with $12.1 \%$ in rural areas (Table 1). In particular, the percentage of new TB cases with MDR-TB in urban areas was $13.5 \%$ as compared to $7.5 \%$ in rural areas. Closer examination of the data in individual cities indicates that the largest cities, Tbilisi and Kutaisi, are the main drivers of this phenomenon (Figure 3). We note that in those districts with higher point estimates of MDR-TB risk than these two cities, the estimates are based on small sample sizes and so have quite limited precision (Figure 3 ).

Individual-level factors associated with multidrug-resistant tuberculosis among tuberculosis cases

Among new TB cases, living in a city ('urban area', Table 3) was statistically significantly associated with being diagnosed with MDR-TB (odds ratio (OR): 1.43; 95\% Cl: 1.20-1.72; p<0.001) compared with living in a rural area. New TB cases aged 35 years and over were 


\section{FIGURE 1}

Average annual incidence of notified multidrug-resistant TB per 100,000 population among new (A) and previously treated (B) TB cases by district/city, Georgia, January 2009-June 2011

A

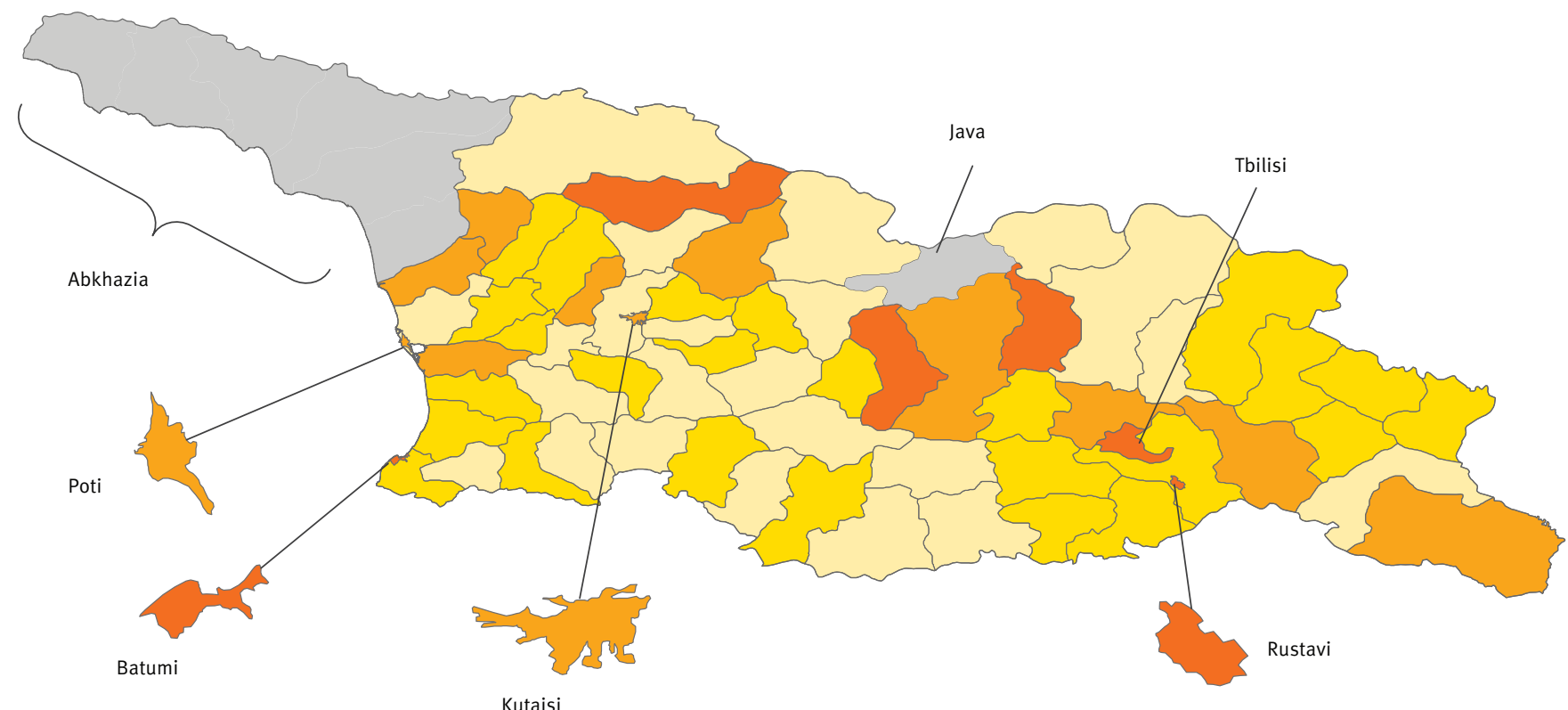

B

MDR-TB incidence per 100,000 population

Kutaisi

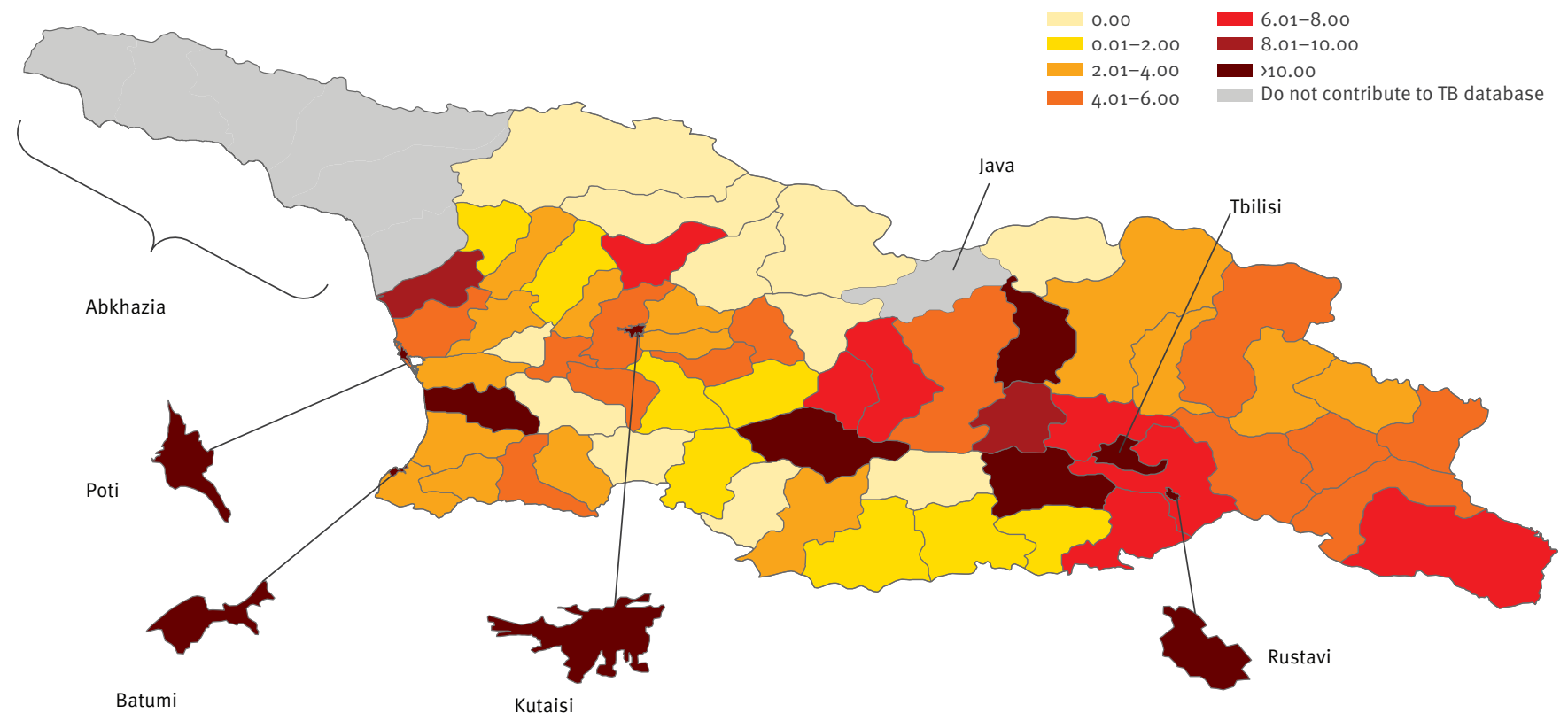

MDR-TB: multidrug-resitant tuberculosis; TB: tuberculosis.

The boundaries of the districts and cities as defined in the National Census [4] are shown. The five areas listed as cities in the National Census are labelled by name and are not shaded in grey. Two areas of Georgia do not report TB cases to the databases analysed: the region of Abkhazia and the district of Java. These two areas are labelled by name and shaded in grey. 


\section{FIGURE 2}

Risk of multidrug-resistant tuberculosis (MDR-TB), as percentage of tuberculosis cases with MDR-TB among new (A) and previously treated tuberculosis cases (B) by district or city, Georgia, January 2009-June 2011

\section{A}

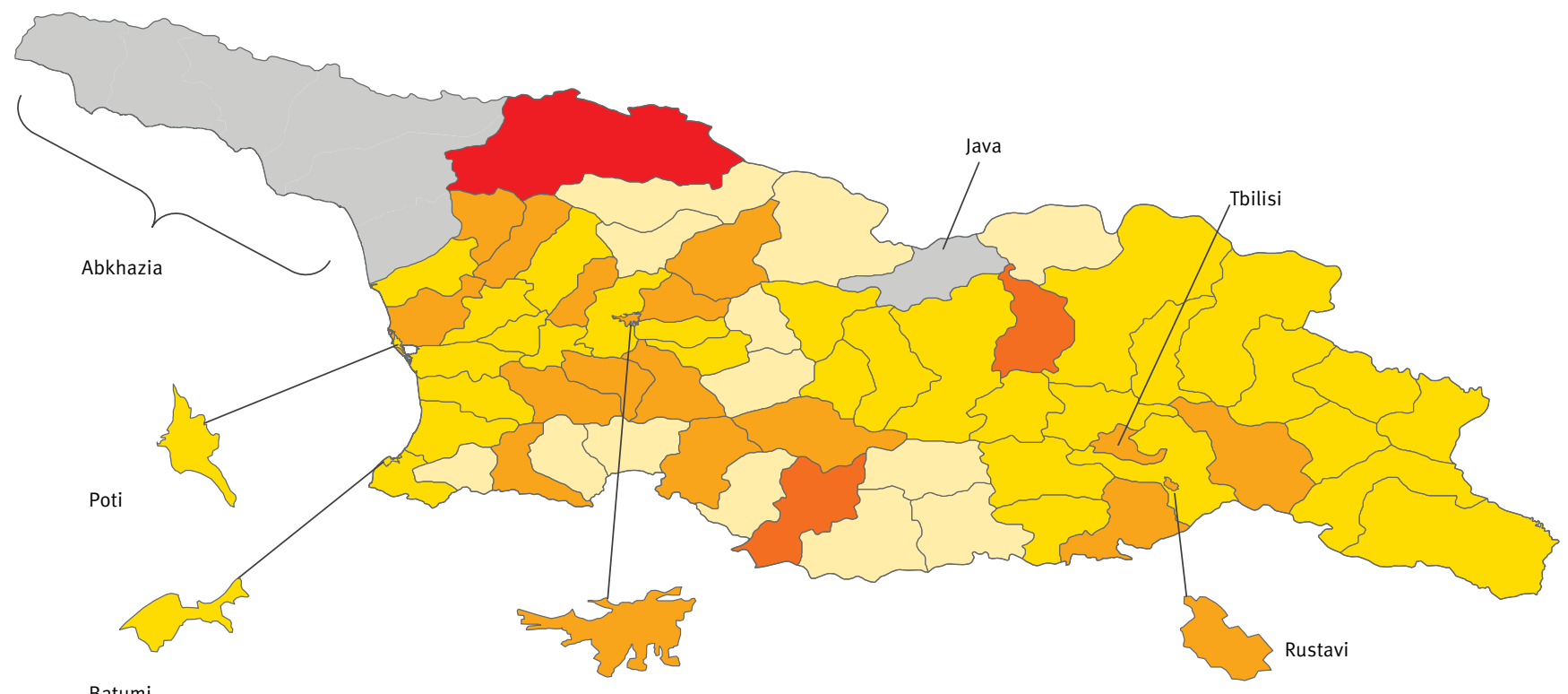

B

Kutaisi

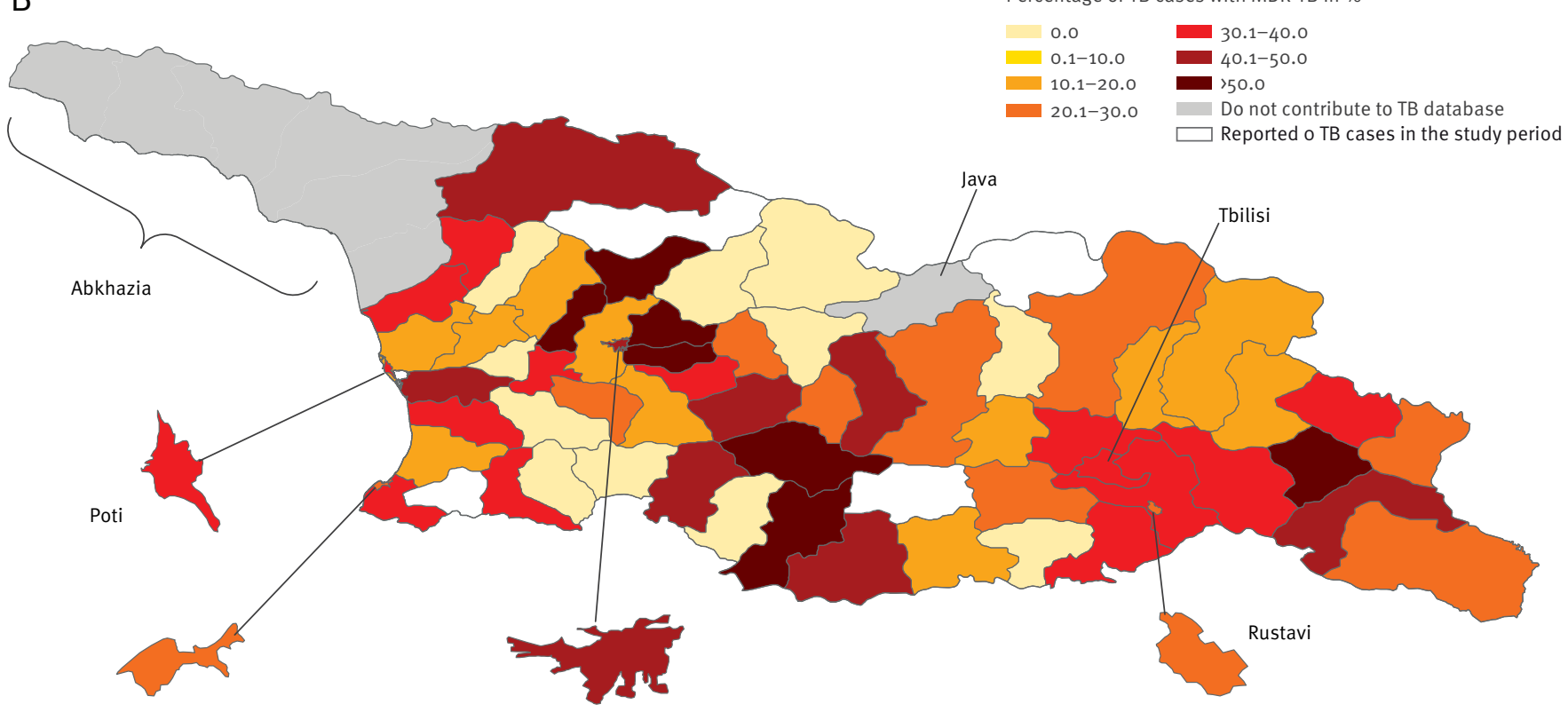

Batumi

Kutaisi

TB: tuberculosis.

The boundaries of the districts and cities as defined in the National Census [4] are shown. The five areas listed as cities in the National

Census are labelled by name and are not shaded in grey. Two areas of Georgia do not report TB cases to the databases analysed: the region of Abkhazia and the district of Java. These two areas are labelled by name and shaded in grey. 


\section{FIGURE 3}

Percentage of notified new (A) and previously treated (B) tuberculosis cases with multidrug-resistant tuberculosis confirmed through drug susceptibility testing by district/city in Georgia, January 2009-June 2011

A

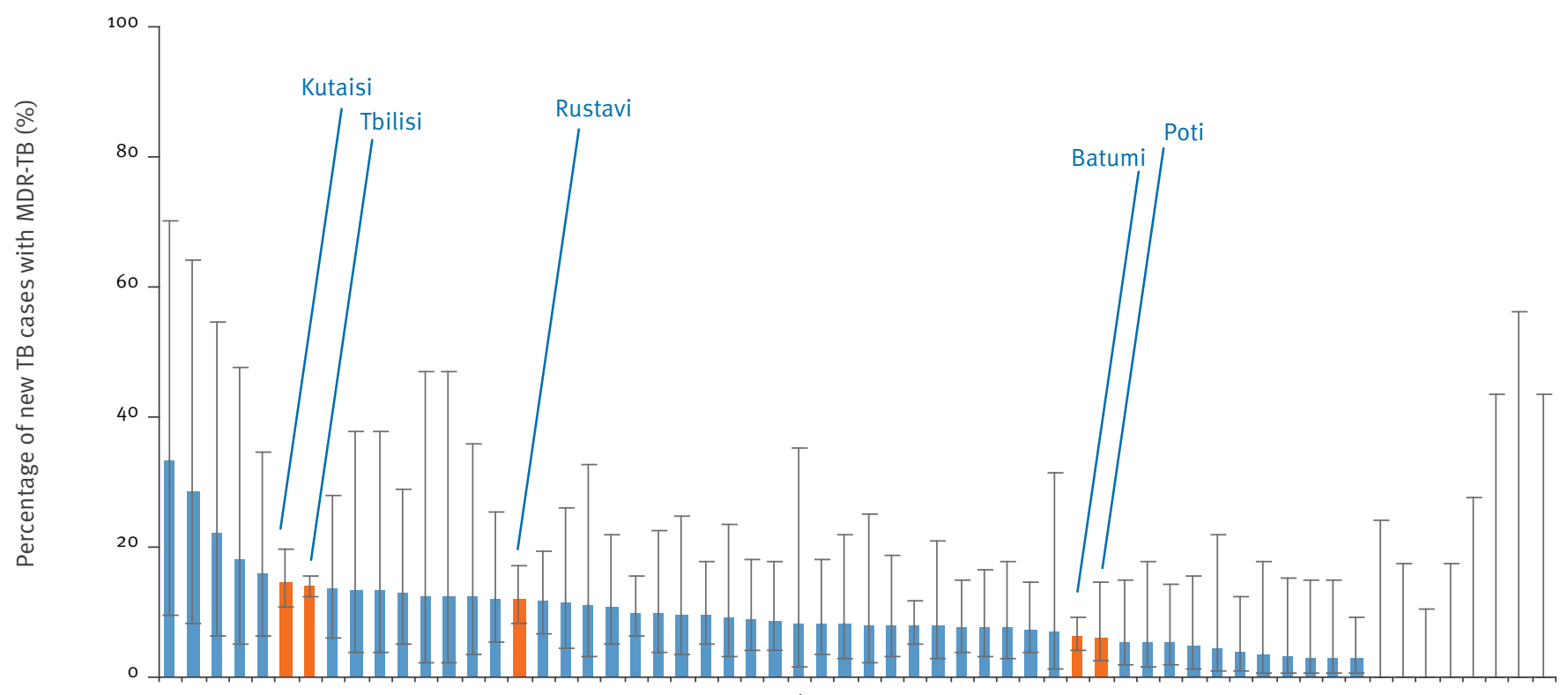

B

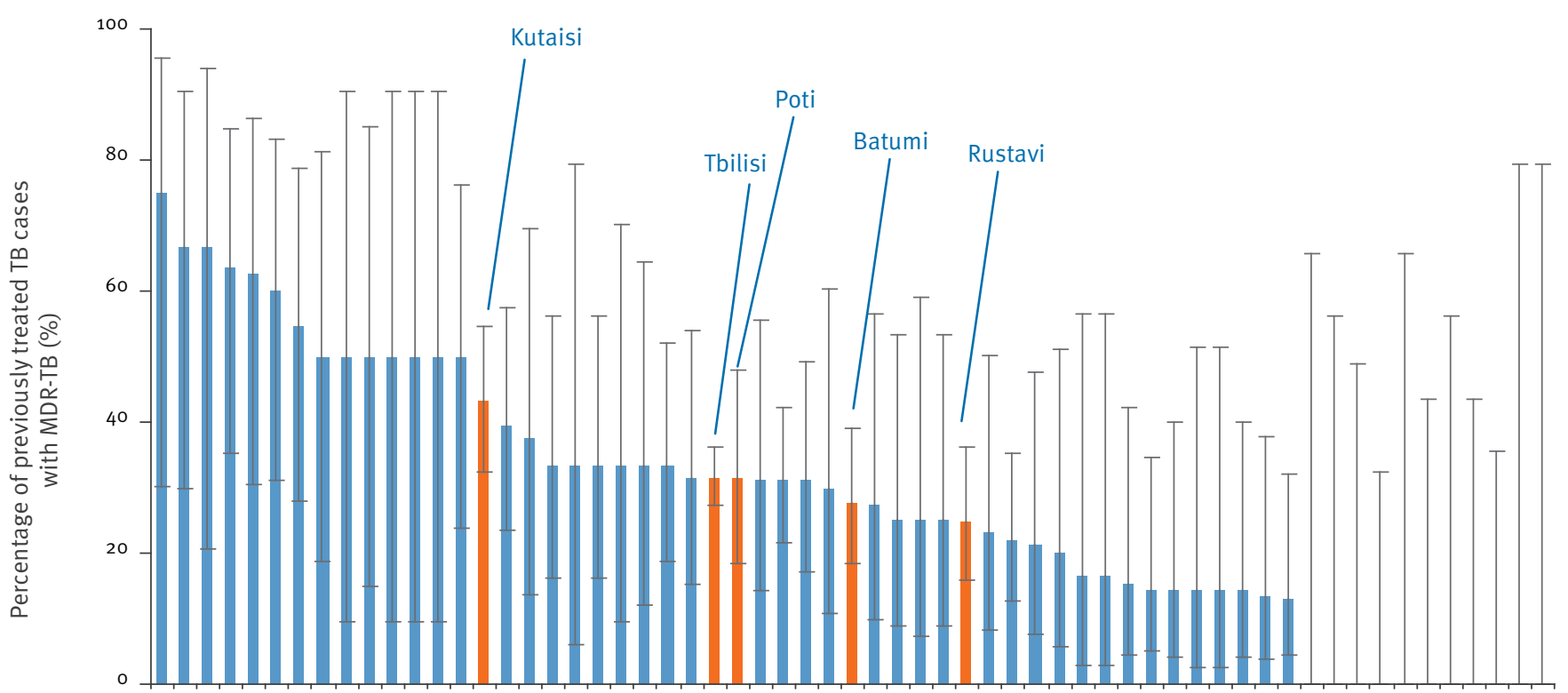

Districts/cities

MDR-TB: multidrug-resistant tuberculosis; TB: tuberculosis.

Each bar represents a different district or city and districts/cities are only included if they reported at least one TB case in the study period. Cities (as defined in the National Census [4]) are shown in red and labelled by name; all other districts are shown in blue. Exact binomial confidence intervals are displayed for each district/city. 


\section{TABLE 3}

Individual-level risk factors for multidrug-resistant tuberculosis diagnosis in new and previously treated tuberculosis cases in Georgia, January 2009-June 2011

\begin{tabular}{|c|c|c|c|c|c|c|c|c|}
\hline \multirow{3}{*}{ Variable } & \multicolumn{4}{|c|}{ New TB cases } & \multicolumn{4}{|c|}{ Previously treated TB cases } \\
\hline & \multicolumn{2}{|c|}{ Univariable model results } & \multicolumn{2}{|c|}{ Multivariable model results } & \multicolumn{2}{|c|}{ Univariable model results } & \multicolumn{2}{|c|}{ Multivariable model results } \\
\hline & $\begin{array}{c}\text { Odds ratio } \\
(95 \% \mathrm{Cl})\end{array}$ & P-value & $\begin{array}{l}\text { Odds ratio } \\
(95 \% \mathrm{Cl})\end{array}$ & P-value & $\begin{array}{l}\text { Odds ratio } \\
(95 \% \mathrm{CI})\end{array}$ & P-value & $\begin{array}{c}\text { Odds ratio } \\
(95 \% \mathrm{Cl})\end{array}$ & P-value \\
\hline \multicolumn{9}{|c|}{ Demographic and socio-economic factors } \\
\hline \multicolumn{9}{|c|}{ Sex } \\
\hline Male & Reference & - & $\mathrm{NI}$ & $\mathrm{NI}$ & Reference & - & Reference & - \\
\hline Female & $1.02(0.86-1.21)$ & 0.81 & $\mathrm{NI}$ & $\mathrm{NI}$ & $0.85(0.68-1.07)$ & 0.14 & $0.93(0.73-1.18)$ & 0.53 \\
\hline \multicolumn{9}{|l|}{ Age (years) } \\
\hline $0-34$ & Reference & - & Reference & - & Reference & - & Reference & - \\
\hline$\geq 35$ & $0.73(0.63-0.84)$ & 0.004 & $0.72(0.62-0.84)$ & $<0.001$ & $0.94(0.82-1.08)$ & 0.37 & $1.09(0.92-1.29)$ & 0.33 \\
\hline \multicolumn{9}{|l|}{ Occupation } \\
\hline Unemployed & Reference & - & $\mathrm{NI}$ & $\mathrm{NI}$ & Reference & - & $\mathrm{NI}$ & $\mathrm{NI}$ \\
\hline Military & $0.34(0.05-2.34)$ & 0.89 & $\mathrm{NI}$ & $\mathrm{NI}$ & Small numbers & - & $\mathrm{NI}$ & $\mathrm{NI}$ \\
\hline Employed & $0.98(0.77-1.25)$ & 0.27 & $\mathrm{NI}$ & $\mathrm{NI}$ & $0.96(0.72-1.27)$ & 0.76 & $\mathrm{NI}$ & $\mathrm{NI}$ \\
\hline \multicolumn{9}{|c|}{ Location at diagnosis } \\
\hline Rural area & Reference & - & Ref & - & Reference & - & $\mathrm{NI}$ & $\mathrm{NI}$ \\
\hline Urban area & $1.43(1.20-1.70)$ & $<0.001$ & $1.43(1.20-1.72)$ & $<0.001$ & $0.92(0.77-1.08)$ & 0.30 & $\mathrm{NI}$ & $\mathrm{NI}$ \\
\hline $\begin{array}{l}\text { Penitentiary } \\
\text { system }\end{array}$ & $1.22(0.98-1.52)$ & 0.074 & $1.20(0.96-1.50)$ & 0.11 & $1.19(1.01-1.40)$ & 0.038 & $\mathrm{NI}$ & $\mathrm{NI}$ \\
\hline \multicolumn{9}{|c|}{ Previously in detention } \\
\hline No & \begin{tabular}{|l|} 
Reference \\
\end{tabular} & - & $\mathrm{NI}$ & $\mathrm{NI}$ & Reference & - & Reference & - \\
\hline Yes & $0.80(0.60-1.07)$ & 0.14 & $\mathrm{NI}$ & $\mathrm{NI}$ & $1.23(1.03-1.46)$ & 0.022 & $1.19(0.99-1.43)$ & 0.071 \\
\hline \multicolumn{9}{|c|}{ Internally displaced person } \\
\hline No & Reference & - & $\mathrm{NI}$ & $\mathrm{NI}$ & Reference & - & $\mathrm{NI}$ & $\mathrm{NI}$ \\
\hline Yes & $0.86(0.55-1.35)$ & 0.50 & $\mathrm{NI}$ & $\mathrm{NI}$ & $0.87(0.57-1.32)$ & 0.49 & $\mathrm{NI}$ & $\mathrm{NI}$ \\
\hline \multicolumn{9}{|l|}{ TB-related factors } \\
\hline \multicolumn{9}{|l|}{ Disease location } \\
\hline Pulmonary & Reference & - & $\mathrm{NI}$ & $\mathrm{NI}$ & Reference & - & $\mathrm{NI}$ & $\mathrm{NI}$ \\
\hline Extra-pulmonary & $1.04(0.77-1.41)$ & 0.79 & $\mathrm{NI}$ & $\mathrm{NI}$ & $1.11(0.74-1.67)$ & 0.63 & $\mathrm{NI}$ & $\mathrm{NI}$ \\
\hline \multicolumn{9}{|c|}{ Smear microscopy result } \\
\hline Negative & Reference & - & Reference & - & Reference & - & $\mathrm{NI}$ & $\mathrm{NI}$ \\
\hline Positive & $1.15(0.94-1.42)$ & 0.18 & $1.21(0.98-1.49)$ & 0.081 & $1.01(0.83-1.23)$ & 0.94 & $\mathrm{NI}$ & $\mathrm{NI}$ \\
\hline Not tested & $2.85(1.39-5.82)$ & 0.004 & $2.91(1.44-5.90)$ & 0.003 & $1.56(0.70-3.48)$ & 0.28 & $\mathrm{NI}$ & $\mathrm{NI}$ \\
\hline \multicolumn{9}{|c|}{ Previous treatment outcome } \\
\hline Cure & NA & NA & NA & NA & Reference & - & $\mathrm{NI}$ & $\mathrm{NI}$ \\
\hline $\begin{array}{l}\text { Completed } \\
\text { treatment }\end{array}$ & NA & NA & NA & NA & $0.92(0.75-1.13)$ & 0.42 & $\mathrm{NI}$ & $\mathrm{NI}$ \\
\hline Default & NA & NA & NA & NA & $0.59(0.46-0.75)$ & $<0.001$ & $\mathrm{NI}$ & $\mathrm{NI}$ \\
\hline Failure & NA & NA & NA & NA & $1.45(1.12-1.87)$ & 0.005 & $\mathrm{NI}$ & $\mathrm{NI}$ \\
\hline Unknown & NA & NA & NA & NA & $0.82(0.57-1.17)$ & 0.27 & $\mathrm{NI}$ & $\mathrm{NI}$ \\
\hline
\end{tabular}

$\mathrm{Cl}$ : confidence interval; NA: not applicable; NI: not included in the multivariable model; TB: tuberculosis.

Cases notified between January 2009 and June 2011 are included.

at a lower risk of MDR-TB (OR: $0.72 ; 95 \% \mathrm{Cl}: 0.62-$ 0.84 ; p 0.001 ). Among previously treated TB cases, those that had previously been in detention may be at a higher risk of MDR-TB (OR: $1.19 ; 95 \% \mathrm{Cl}$ : 0.99-1.43; $\mathrm{p}=0.071$ ).

\section{Discussion}

We found marked geographical heterogeneity in both MDR-TB incidence and risk. Identification of such 'hot-spots' of MDR-TB disease is important in order to appropriately allocate resources and to identify locations for further studies that might inform interventions aimed at reducing the MDR-TB incidence in 
Georgia. The variation in both the incidence of MDR-TB per 100,000 population and the percentage of TB cases with MDR-TB between districts was considerable with some districts reporting a disease incidence or risk several times higher than that of other districts (up to 23-fold for MDR-TB incidence and up to 12 -fold for MDR-TB risk). This adds further evidence to the literature indicating that such MDR-TB 'hot-spots' may be common to high burden countries $[6,12]$.

Most notably, we found that the risk of MDR-TB among new TB cases was higher in the cities than in rural areas even when adjusting for other potentially confounding factors such as age. Since new TB cases are those that have received either no (or at most one month of) TB treatment previously, the new TB cases with MDR-TB are likely to have been infected with resistant strains. Thus, these results suggest that these urban areas, especially the two largest cities, are where the risk of transmitted MDR-TB is relatively high compared with other less-resistant forms of TB (Table 1). Our results are consistent with a previous study in Georgia that found that living in Tbilisi (the capital city) was a risk factor for MDR-TB [13]. It should be noted that the definitions of urban and rural may overlap and areas on the outskirts of cities may be more similar to rural areas in many respects than they are to the centres of cities.

We found that $10 \%$ and $33 \%$ of new and previously treated TB cases respectively had MDR-TB. These estimates are nearly identical to those that Georgia reported to the WHO for 2011 although they are slightly lower than estimates reported in previous studies $[1,13,14]$. These percentages are worrying and within the range that has been reported from other countries of the eastern part of the WHO European Region [3].

Notified MDR-TB incidence in the penitentiary system was 837 per 100,000 people. This is worryingly high and around 50 times the rate estimated for the nationwide population. Young men are overrepresented in prison populations and because this demographic group is likely at greater than average risk of TB and MDR-TB regardless of incarceration status, it is not surprising that MDR-TB is concentrated in prisons. However, even if we assume that the penitentiary system is composed entirely of men aged between 25 and 34 years, we would still only expect a notified MDR-TB incidence of $56 / 100,000$ (Table 2 ) if the rates of MDR-TB were similar to that of the civilian sector. This is only one fifteenth of the rate that we actually found in the prisons. It is also possible that screening among prisoners at time of entry could partially account for the increased notifications in this setting. However, since only around $10 \%$ of TB cases diagnosed in prison are found at entry (M. Gegia, personnal communication, 14 May 2013) we think entry screening is unlikely to account for the entire excess burden observed in the prisons. High MDR-TB incidence in the penitentiary system of the order of magnitude found in this study has also been identified previously in Georgia [15] and in Moldova [6] (also in the eastern part of the WHO European Region). The percentage of new TB cases with MDR-TB in the penitentiary system is nearly identical to that in the rest of the country indicating that the high MDR-TB incidence in that setting is driven by increased TB risk rather than a specific risk of transmitted MDR-TB. However, increased MDR-TB risk among TB cases in the penitentiary system has been found previously within Georgia and elsewhere in the eastern part of the WHO European Region, for example, in Russia and Ukraine [16-18].

Potential limitations of our study include those commonly found when using routinely collected surveillance data. Theoretically, the identification of substantial geographical heterogeneity in MDR-TB incidence in a setting may be attributable to variable case finding and reporting of TB and variable culture and DST use in MDR-TB diagnosis. However, Georgia is a small country with a relatively well-structured TB diagnosis and testing system; variability in case detection across the country is likely to be minimal and culture and DST are respectively required (under national policy) for all TB suspects and culture positive cases. Despite the completeness of this dataset compared to the majority of TB surveillance datasets from high burden countries, bias was potentially introduced by the absence of one third of culture and DST results from the TB notifications database. However, examination of the percentage of missing data from subgroups in the data indicated that there was no systematic bias that would have substantially affected our results. For example, data on culture results were missing from $32 \%$ and $33 \%$ of urban and rural cases respectively, $32 \%$ and $39 \%$ from male and female cases respectively and between $30 \%$ and $39 \%$ from each age class, other than those under 15 years where we would expect greater percentages missing due to difficulties with obtaining specimens. In this study, we attributed each case to a district; we recommend that future studies be conducted that allow the collection of global positioning system (GPS) coordinates of residential locations to allow for more refined spatial analysis, particularly in urban settings.

Aside from a marked difference between urban and rural areas, the underlying reasons for the spatial heterogeneity in MDR-TB are unclear. We attempted to obtain further information on potential explanatory factors such as average household income or proportion of the population in a district that are unemployed from the National Census. However, these data were only available at the national level rather than the district level and without data for each district, we could not investigate associated factors statistically. Understanding these patterns of spread of MDR-TB, which appear to be most severe within urban settings and penitentiaries, requires more refined geographical and genotypic data. Our analysis, which is limited to routinely available data has highlighted important 
heterogeneities but leaves many questions about the causes of this variability unanswered.

We have identified substantial geographic heterogeneity in both the risk and incidence of MDR-TB in Georgia, a pattern that may be commonly found in countries in the eastern part of the WHO European Region. Such analysis is crucial to determine where to target resources and prioritise areas for further studies and interventions aimed at reducing the considerable MDR-TB prevalence in these high burden countries.

\section{Acknowledgments}

We thank all those involved in surveillance, laboratory testing and treatment for tuberculosis in the Republic of Georgia. The project described was supported by Award Number U54GMo88558 and DP2ODo06663 from the National Institute of General Medical Sciences and by Award Number Ko1Al102944 from the National Institute of Allergy and Infectious Diseases. The content is solely the responsibility of the authors and does not necessarily represent the official views of the National Institute of General Medical Sciences or the National Institute of Allergy and Infectious Diseases or the Office of the Director, National Institutes of Health. The funders had no role in study design, data collection and analysis, decision to publish, or preparation of the manuscript. MG and TCh were supported by the NIH/Fogarty International Center grant D4TWo07124.

\section{Conflict of interest}

None declared.

\section{Authors' contributions}

HEJ and TCo conceived and designed the study. UN and TCh collected the data. HEJ analysed the data. MG, JF and IK helped interpret the results. HEJ and TCo wrote the first draft of the manuscript. All authors contributed to revising the manuscript and read and approved the final version.
7. Cohen T, Manjourides J, Hedt-Gauthier B. Linking surveillance with action against drug-resistant tuberculosis. Am J Respir Crit Care Med. 2012;186(5):399-401 http://dx.doi.org/10.1164/rccm.201203-0394PP

8. Manjourides J, Lin HH, Shin S, Jeffery C, Contreras C, Cruz JS, et al. Identifying multidrug resistant tuberculosis transmission hotspots using routinely collected data. Tuberculosis (Edinb). 2012;92(3):273-9. http://dx.doi.org/10.1016/j.tube.2012.02.003

9. Heifets L. Conventional methods for antimicrobial susceptibility testing of Mycobacterium tuberculosis. In: Bastian I, Portaels F, editors. Multidrug-resistant tuberculosis. Dordrecht: Springer Netherlands; 2000. p. 133-43. http://dx.doi.org/10.1007/978-94-011-4084-3_8

10. World Health Organization (WHO). Treatment of tuberculosis guidelines, fourth edition. Geneva: WHO; 2010. [Accessed 9 May 2013]. Report No.: WHO/HTM/TB/2009.420.

11. World Health Organization (WHO). Guidelines for drug resistance surveillance in tuberculosis, 4 th edition. Geneva WHO; 2009. [Accessed 9 May 2013]. Report No.: WHO/HTM/ $\mathrm{TB} / 2009.422$.

12. Lin H, Shin S, Blaya JA, Zhang Z, Cegielski P, Contreras C, et al. Assessing spatiotemporal patterns of multidrug-resistant and drug-sensitive tuberculosis in a South American setting. Epidemiol Infect. 2011;139(11):1784-93. http://dx.doi.org/10.1017/S0950268810002797

13. Vashakidze L, Salakaia A, Shubladze N, Cynamon M, Barbakadze K, Kikvidze M, et al. Prevalence and risk factors for drug resistance among hospitalized tuberculosis patients in Georgia. Int J Tuberc Lung Dis. 2009;13(9):1148-53.

14. Mdivani N, Zangaladze E, Volkova N, Kourbatova E, Jibuti T, Shubladze N, et al. High prevalence of multidrug-resistant tuberculosis in Georgia. Int J Infect Dis. 2008;12(6):635-44. http://dx.doi.org/10.1016/j.ijid.2008.03.012

15. Aerts A, Habouzit M, Mschiladze L, Malakmadze N, Sadradze $\mathrm{N}$, Menteshashvili $\mathrm{O}$, et al. Pulmonary tuberculosis in prisons of the ex-USSR state Georgia: results of a nation-wide prevalence survey among sentenced inmates. Int J Tuberc Lung Dis. 2000;4(12):1104-10.

16. Ruddy M, Balabanova Y, Graham C, Fedorin I, Malomanova $\mathrm{N}$, Elisarova $\mathrm{E}$, et al. Rates of drug resistance and risk factor analysis in civilian and prison patients with tuberculosis in Samara Region, Russia. Thorax. 2005;60(2):130-5. http://dx.doi.org/10.1136/thx.2004.026922

17. Dubrovina I, Miskinis K, Lyepshina S, Yann Y, Hoffmann H, Zaleskis R, et al. Drug-resistant tuberculosis and HIV in Ukraine: a threatening convergence of two epidemics? Int Tuberc Lung Dis. 2008;12(7):756-62.

18. Jugheli L, Rigouts L, Shamputa IC, Bram de Rijk W, Portaels F. High levels of resistance to second-line anti-tuberculosis drugs among prisoners with pulmonary tuberculosis in Georgia. Int J Tuberc Lung Dis. 2008;12(5):561-6.

\section{References}

1. World Health Organization (WHO). Global Tuberculosis Report 2012. Geneva: WHO; 2012. [Accessed 9 May 2013]. Report No.: WHO/HTM/TB/2012.6.

2. Orenstein EW, Basu S, Shah NS, Andrews JR, Friedland GH, Moll AP, et al. Treatment outcomes among patients with multidrug-resistant tuberculosis: systematic review and metaanalysis. Lancet Infect Dis. 2009;9(3):153-61. http://dx.doi.org/10.1016/S1473-3099(09)70041-6

3. Zignol M, van Gemert W, Falzon D, Sismanidis C, Glaziou P, Floyd K, et al. Surveillance of anti-tuberculosis drug resistance in the world: an updated analysis, 2007-2010. Bull World Health Organ. 2012;90(2):111-9D. http://dx.doi.org/10.2471/BLT.11.092585

4. National Statistics Office of Georgia. National Census Data. [Accessed 9 May 2013]. Available from: http://geostat.ge/ index.php?action=page\&p_id=674\&lang=eng

5. Furin J, Gegia M, Mitnick C, Rich M, Shin S, Becerra M, et al. Eliminating the category II retreatment regimen from national tuberculosis programme guidelines: the Georgian experience. Bull World Health Organ. 2012;90(1):63-6. http://dx.doi.org/10.2471/BLT.11.092320

6. Jenkins HE, Plesca V, Ciobanu A, Crudu V, Galusca I, Soltan $\mathrm{V}$, et al. Assessing spatial heterogeneity of multidrugresistant tuberculosis in a high-burden country. Eur Respir J. 2013;42(5):1291-301.

http://dx.doi.org/10.1183/09031936.00111812 